\title{
High Pressure Effects on the Structural Properties of GaN Compound Using Equations of State
}

\author{
Siham J. AL-Faris ${ }^{1 *}$, Raed H. Al-Saqa ${ }^{2}$, Huda M. Mohmad ${ }^{3}$, Sirwan K. Jalal ${ }^{4}$ \\ ${ }^{1 *}$ Alnoor University College Bartella ,Iraq \\ ${ }^{2}$ Directorate General of Education, Iraq \\ ${ }^{3}$ Alnoor University College Bartella ,Iraq \\ ${ }^{4} \mathrm{G}$. Science Department, Charmo University, Iraq \\ E-mail: ${ }^{1 *}$ siham.jasim@alnoor.edu.iq
}

Received 1 July 2021, Revised 13 October 2021, Accepted 2 November 2021

\begin{abstract}
The present study is a theoretical calculation for the effects of high pressure on thermodynamic properties on GaN up to $40 \mathrm{Gpa}$ at room temperature. Volume compression ratio $\left(\mathrm{V}_{0} / \mathrm{V}_{\mathrm{p}}\right)$, lattice constant (a) and elastic bulk modulus(B) have been established. Furthermore, lattice frequencies and disruptions function by analyzing phonon frequency spectrum (PFS) at $(0 \mathrm{~K})$. The entire calculations rely on using of two equation of state (EOS) "BirchMurnaghan and modified Lennard-Jones" equation of state and with the integration of Grüneisen approximation theory. From the considered equations of state, formulation of bulk modulus was derived, that predicts a rising trend of bulk modulus. The large bulk modulus value of $\mathrm{GaN}$ has made a small fraction of change in volume (less than $15 \%$ ) of the material even under an extreme pressure up to $45 \mathrm{Gpa}$. It was also found that the results of phonon frequency spectrum obtained from Birch-Murnaghan equation of state in a better agreement with the experimental data than that of modified Lennard-Jones equation of state. Given that the Birch-Murnaghan equation of state developed according to Eulerian strain theory accounted as a universal equation of state. Moreover, good agreement between theoretically present calculations and experiment data of phonon frequency spectrum, reveals the validity of the equations of state used in the present study.
\end{abstract}

Keywords: Bulk modulus; volume compression ratio; lattice parameter; phonon frequency spectrum.

\section{Introduction}

The semiconductor materials represent today's basic building blocks of emitters and receivers in cellular, satellite, and fiberglass communications. III-nitrides, for example, are nowadays the most widely used type of semiconducting materials in the industry [1]. The III-V nitride: GaN has exhibited particular interest due to some of its attracting properties such as large energy gap, high thermal conductivity, large bulk modulus and the extreme hardness. In addition, GaN has high melting point. These, characteristics that are closely related to their strong (Ionic and covalent) bonding, they make the material very promising for optoelectronic device applications [2]. The zinc blende structured $\mathrm{GaN}$ has a higher saturated electron drift velocity and a somewhat lower energy gap than GaN [3]. Vibrational contribution of lattice frequencies are represented by phonon frequency spectrum, which is found to be volume or pressure dependent, then volume dependent of vibrational modes are characterized by Grüneisen parameter.

High pressure research have been an interesting field of condensed matter. Pressure induces vital structural properties within crystalline solids, for example as high pressure is applied, bulk modulus tends to increase, the Grüneisen parameter declines and also phonon frequency spectrum alters [4,5]. As high pressure alters mechanical and thermodynamic properties of solid crystalline, thus high pressure can induce new structural materials with useful characterization.

Theoretical condensed matter research has developed equations of state to predict numerically interesting properties of material, for example, thermal-pressure equation of state which demonstrates the thermally generated pressure due to lattice vibrations [6] and isothermal equation of state which is the main approach in the current study.

In the present work, the bulk modulus (B), compression volume $(\mathrm{VP} / \mathrm{Vo})$, lattice constant and phonon frequency spectrum (pfs) of a GaN compound under the influence of high pressure were calculated using the "Birch-Murnaghan and modified Lennard-Jouns" EOS. The calculated results have been compared with experimental data, which confirms the validity of the present equations of state. The Grüneisen parameter variation assumption has improved the results of phonon frequency spectrum under compression.

\section{Theoretical Details}

Equation of state (EOS) of crystalline materials is a straight forward mathematical expression relating high pressure $\mathrm{P}$ applied to compress the solid isothermally from initial volume $\mathrm{V}_{0}$ to $\mathrm{V}$. Thus, equation of state is analogue to general gas equation $\mathrm{PV}=\mathrm{nRT}$. EOSs are cost effective and time saving method, though which and without any laboratory, various outstanding properties of solid phase 
can be found. Depending on various assumptions, variety of EOS have been developed in the literature. Current study focuses on using of two familiar equations that are presented in the following sections:

\subsection{Birch-Murnaghan equations of state (B-M EOS)}

Eulerian strain represents the strain relative to the strained state, it has widespread applications for understanding high pressure behavior of solid matter.

The Eulerian strain $\left(f_{e}\right)$ is given by:

$$
f_{e}=\frac{1}{2}\left[\left(\frac{V_{0}}{V_{P}}\right)^{2 / 3}-1\right]
$$

where $V_{0}$ is the volume at atmosphere pressure and $V_{\mathrm{p}}$ is the volume at pressure $\mathrm{P}$.

The B.M EOS is obtained by expanding a series of powers of the Eulerian strain. The 2nd, 3rd and 4th order isothermal B.M EOS, they are functions of two measurable parameters, and isothermal bulk modulus $\left(\mathrm{B}_{0}\right)$ at $\mathrm{P}=0$, so that $\mathrm{P}=\mathrm{f}\left(\mathrm{X}, \mathrm{B}_{0}\right)$, where $\mathrm{X}=\mathrm{V}_{0} / \mathrm{V}_{\mathrm{p}}$. The $\mathrm{B}-\mathrm{M}$ EOS at the 2 nd order when it varies as function of pressure is [7]:

$$
P_{\text {B-M }}=\frac{3 \mathrm{~B}_{0}}{2}\left(\eta^{-7 / 3}-\eta^{-5 / 3}\right)\left(1+\frac{3}{4}\left(\mathrm{~B}_{\mathrm{O}}^{\prime}-4\right)\left(\eta^{-2 / 3}-1\right)\right)
$$

where $\eta$ denotes $\mathrm{V}_{\mathrm{p}} / \mathrm{V}_{\mathrm{o}}$.

2.2 Modified Lennard-Jones equation of state (mL-J EOS)

This equation which proposed two-parameter EOS based on the generalized Lennard-Jones (GLJ) potential which given by $[8]$ :

$$
\mathrm{P}=\frac{B_{0}}{n}\left(V_{0} / V_{P}\right)^{n}\left[\left(V_{0} / V_{P}\right)^{(2 / 3)}-1\right]
$$

where, $n=\frac{1}{3} B_{0}^{\prime}$

$B_{0}^{\prime}$ : First pressure derivative of bulk modulus, and mathematically $B_{0}^{\prime}=\left(\frac{\partial B}{\partial P}\right)_{T}$. Where B is bulk modulus. ML-J EOS is just a two-parameter EOS, and the precision is higher than those for several popular EOSs.

\subsection{Bulk modulus}

The bulk modulus which is a physical constant of solid that indicates their properties when they are under pressure over their entire surfaces is defined as:

$$
B=-\frac{\partial P}{\partial V}
$$

From derivation of equations (2 and 3 ) with respect to volume and substitute them into the equation (4), expressions of pressure dependence of bulk modulus are formulated as given in eqs.5 and 6:
$B_{B-M}=\frac{3 B_{o}}{2}\left[\begin{array}{l}\frac{7}{3} \eta^{-7 / 3}-\frac{5}{3} \eta^{-5 / 3}-\frac{9}{4}\left(B_{o}^{\prime}-4\right) \eta^{-3} \\ +\frac{7}{2}\left(B_{o}^{\prime}-4\right) \eta^{-7 / 3}+\frac{5}{4}\left(B_{o}^{\prime}-4\right) \eta^{-5 / 3}\end{array}\right]$

$B_{(m L-J)}=B_{o}\left(V_{o} / V_{P}\right)^{n}\left[2\left(V_{o} / V_{P}\right)^{n}-1\right]$

\subsection{Lattice constant}

Lattice constant change with pressure was calculated using equation $(7)[9,10]$ :

$a_{p}=a_{o}\left(1+B_{0}^{\prime} \frac{P}{B_{0}}\right)^{-\frac{1}{3 B_{0}^{\prime}}}$

where $a_{0}$, is lattice parameter at ambient condition.

$a_{\mathrm{p}}$, is lattice parameter under compression.

According to Murnaghan EOS [11] the expression $\left(1+B_{0}^{\prime} \frac{P}{B_{o}}\right)^{-1 / B_{0}^{\prime}}$ represents $\left(\frac{V_{P}}{V_{o}}\right)$, Then eq. (7) is written as:

$a_{P}=a_{0}\left(\frac{V_{P}}{V_{o}}\right)^{-\frac{1}{3}}$

\section{Calculation and Results}

\subsection{Evaluation of $V_{P} / V_{0}$ of $G a N$}

The compressibility of $\mathrm{GaN}$ was calculated using the B$\mathrm{M}$ and $\mathrm{mL-J}$ equation of state (eq.2 and 3) show in Fig. 1. In which the input parameters are; $B_{0}$ and $B_{0}^{\prime}$, listed in Table 1. Under the application of high pressure, the material compresses and the volume of the unit cells tends to shrink as expected by the EOSs and seen in the Fig.1. Due to the high bulk modulus $B_{o}(200 \mathrm{Gpa})$ of GaN, $45 \mathrm{Gpa}$ of pressure is required to reduce the volume of the sample to $85 \%$ of its initial value. The $\mathrm{V}_{\mathrm{P}} / \mathrm{V}_{\mathrm{o}}$ curves obtained with B-M EOS and ML-J EOS are inline so that no divergence is observed.

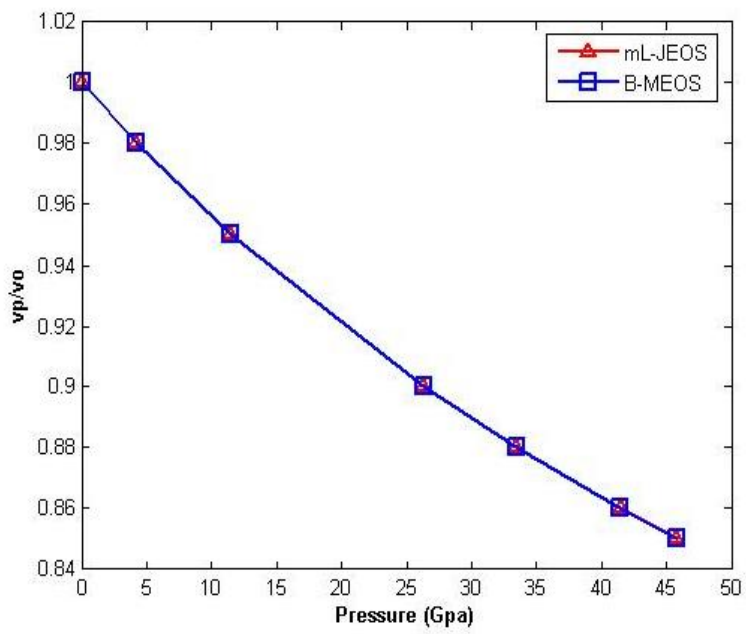

Figure 1. Variation of compressibility with pressure. 
Table 1. Values of GaN parameters, at atmosphere pressure and room temperature

\begin{tabular}{lrl}
\hline Parameters & Values & References \\
\hline$B$ o & 200Gpa & {$[12]$} \\
$B_{0}^{\prime}$ & 4.4 & {$[12]$} \\
$a_{0}$ & $4.5 \AA$ & {$[13]$} \\
$\gamma_{\mathrm{o}}$ & 1.17 & {$[14]$} \\
\hline
\end{tabular}

\subsection{Evaluation of Bulk modulus}

Equations (5 \& 6) represent linear increase in bulk modulus of solid material with reducing volume. Substituting parameters in Table 1 into eqs. $(5 \& 6)$ variation of bulk modulus is calculated and depicted in fig.2. Both EOSs predict that as applied pressure reaches $45 \mathrm{Gpa}$ or $\mathrm{V}_{\mathrm{p}} / \mathrm{V}_{\mathrm{o}}=0.85$, bulk modulus grows to an enormous value as $380 \mathrm{Gpa}$.

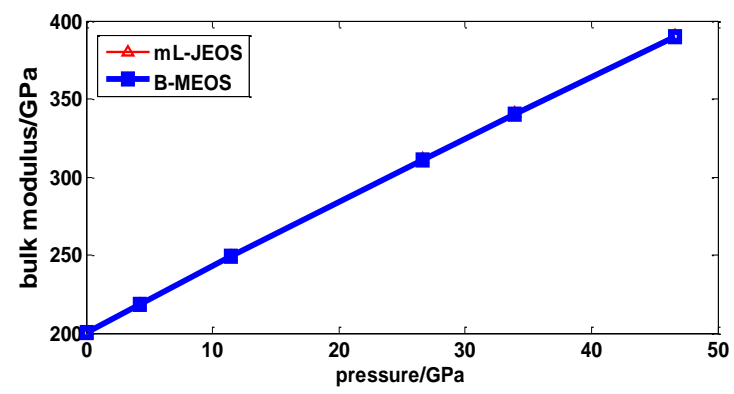

Figure 2. Variation of Bulk modulus with pressure according to the two EOSs.

Moreover, the effect of pressure on lattice parameter has been demonstrated in fig.3 with implement of equation (8), as shown in the following.

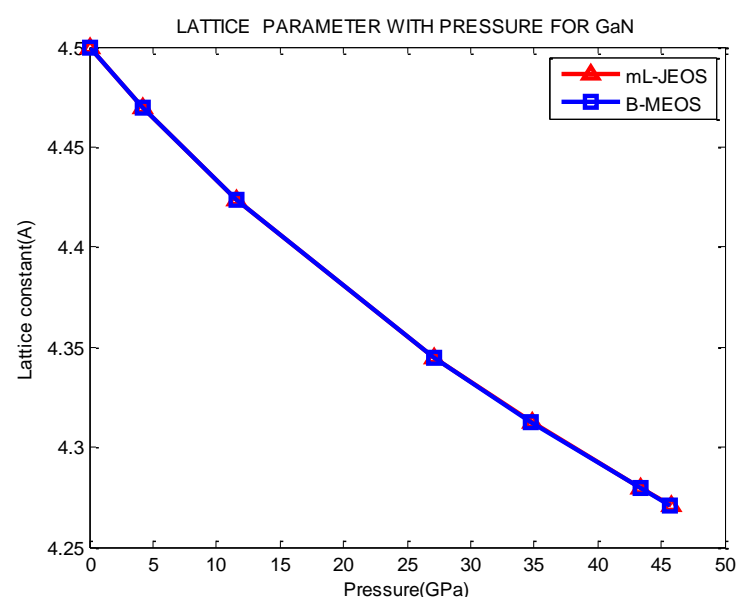

Figure 3. Variation of lattice parameter with pressure according to the two EOSs.

3.3 Phonon frequency spectrum under high pressure pfs

The high pressure produce change in $\left(\mathrm{V}_{\mathrm{p}} / \mathrm{V}_{\mathrm{o}}\right)$ ratio, where high pressure changes the equilibrium position of lattice points and then produces the change in "pfs" as well [15], the equation that describe the "pfs" under high pressure is given by [13]:

$v_{P}=v_{0}\left(\frac{V_{P}}{V_{0}}\right)^{-\gamma}$
$g_{P}\left(v_{P}, V_{P}\right)=g_{0}\left(v_{0}, V_{0}\right)\left(\frac{V_{P}}{V_{0}}\right)^{-\gamma}$

where $v_{\mathrm{p}}$ : frequency at pressure $(\mathrm{P})$.

$v_{0}$ : Frequency at atmospheric pressure.

$\gamma_{0}$ : Grüneisen parameter at atmospheric pressure.

$\mathrm{G}_{\mathrm{P}}\left(v_{\mathrm{P}}, \mathrm{V}_{\mathrm{P}}\right):$ Phonon density of state at pressure $(\mathrm{p})$.

$\mathrm{g}\left(v_{0}, V_{0}\right)$ :Phonon density of state at atmospheric pressure.

\subsection{Evaluation of PFS for GaN under high pressure}

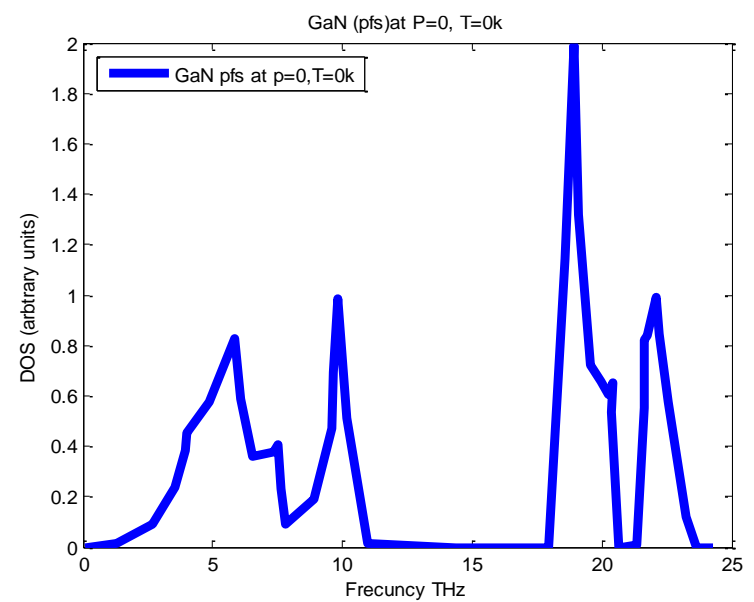

Figure 4. phonon frequency spectrum for GaN at atmospheric pressure and ' $0 K^{\prime}$ [16]

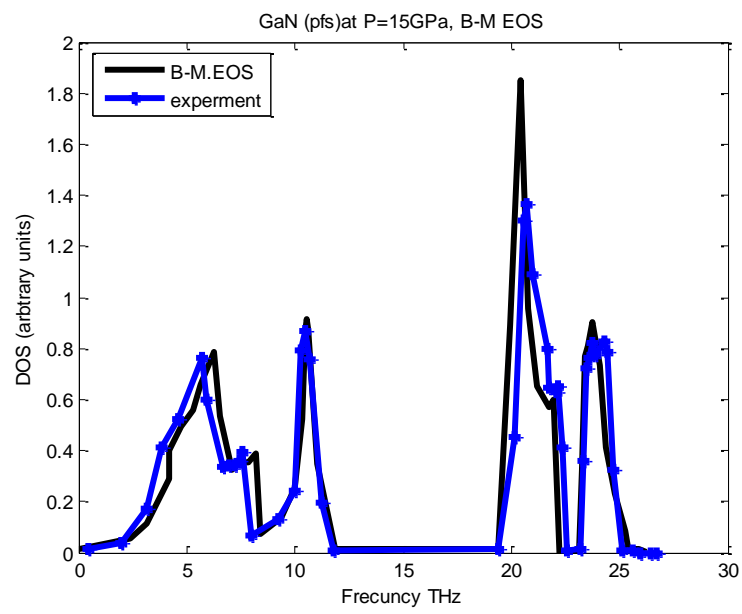

(a)

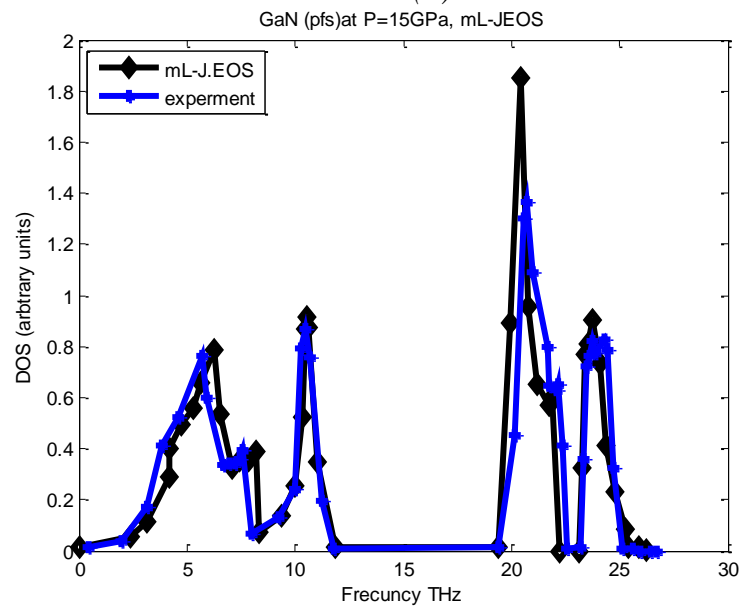

(b)

Figure 5. phonon frequency spectrum for GaN under pressure $P=15 \quad G P a$ using a- B-MEOS b-mL-JEOS compared with [16]. 
Figure (4) represents 'pfs ' for GaN at atmospheric pressure and ' $0 \mathrm{~K}$ '. This section involves calculation of the effect of high pressure on pfs at different values of pressure $15 \mathrm{Gpa}$ and $30 \mathrm{Gpa}$, using two equation of state "B-M EOS and mL-J EOS".

Given that value of $\left(\mathrm{V}_{\mathrm{P}} / \mathrm{V}_{\mathrm{o}}\right)$ under pressures $(15 \mathrm{Gpa}$ and 30Gpa), has already known in fig. 1 . Then combing the data of fig.4 with eqs. (9 and 12), the results for variation of "pfs" for GaN under high pressure using two equations" BMEOS and mL-JEOS" has obtained and shown in figs. (56).

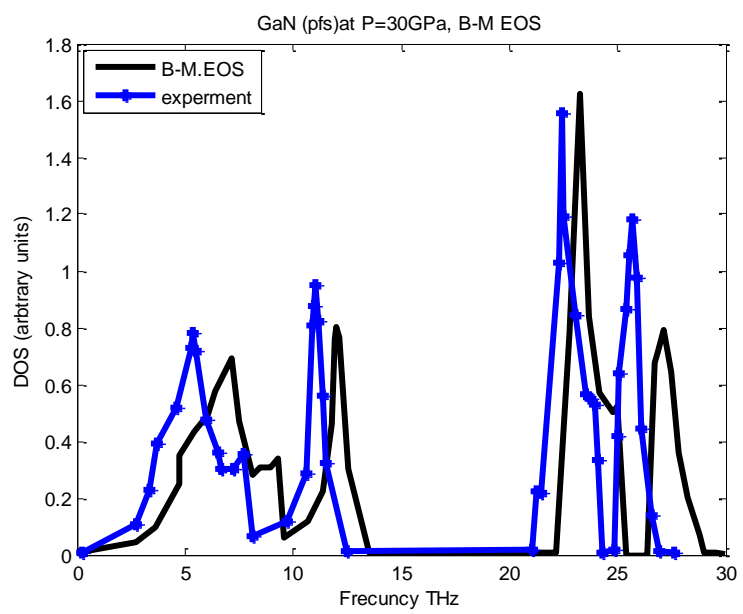

(a)

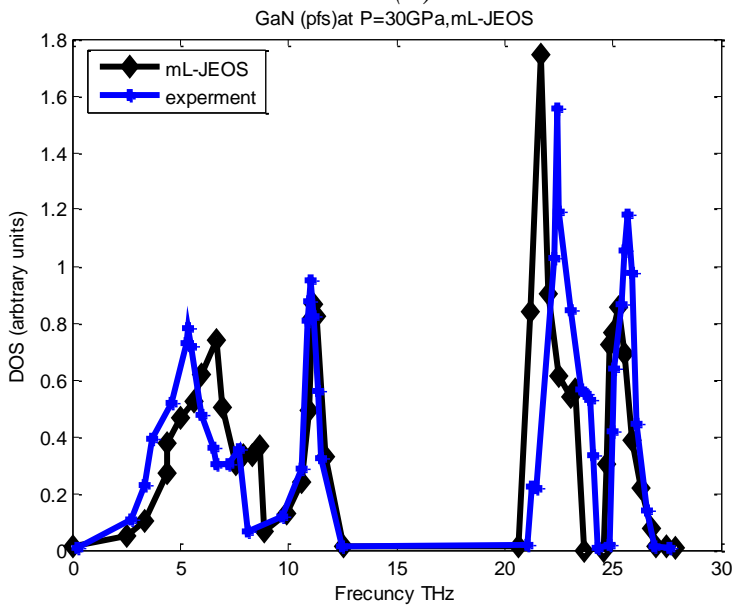

(b)

Figure 6. phonon frequency spectrum for GaN under pressure $P=30 G P a$ using (a) B-MEOS (b) $m L-J$ EOS, compared with experimental data [16].

\section{Grüneisen parameter}

"The Grüneisen parameter $(\gamma)$ is of considerable importance to earth scientist because it sets limitations on the thermo elastic properties of the lower core and mantle [16]. It is dimensionless and used for wide range of solid, it has an approximately constant value and varying slowly with high pressure [17]as will be seen later in fig.6.

\subsection{Grüneisen parameter under high pressure}

The microscopic definition of Grüneisen parameter describes the vibrational motion of atoms. As the oscillation of atoms changes with high pressure so does the Grüneisen parameter. High pressure dependence of Grüneisen parameter is expressed by the following relation [19]:
$\gamma_{P}=\gamma_{0}\left(\frac{V_{P}}{V_{0}}\right)^{q}$

where $\gamma_{0}$ : Grüneisen parameter at atmospheric pressure. $\gamma_{P}:$ Grüneisen parameter under high pressure.

$\mathrm{q}$ : Second Grüneisen parameter, $\mathrm{q}$ has been considered to equal unity.

Using $\gamma_{\mathrm{o}}$ value in table 1 and combining $\mathrm{V}_{\mathrm{P}} / \mathrm{V}_{\mathrm{O}}$ value from fig. 2 in to eq. (11), we get $\gamma_{P}$ which declines slowly with increasing pressure. As it can be observed that even at the highest pressure $(45 \mathrm{Gpa})$, the Grüneisen parameter is reduced to approximately 1 .

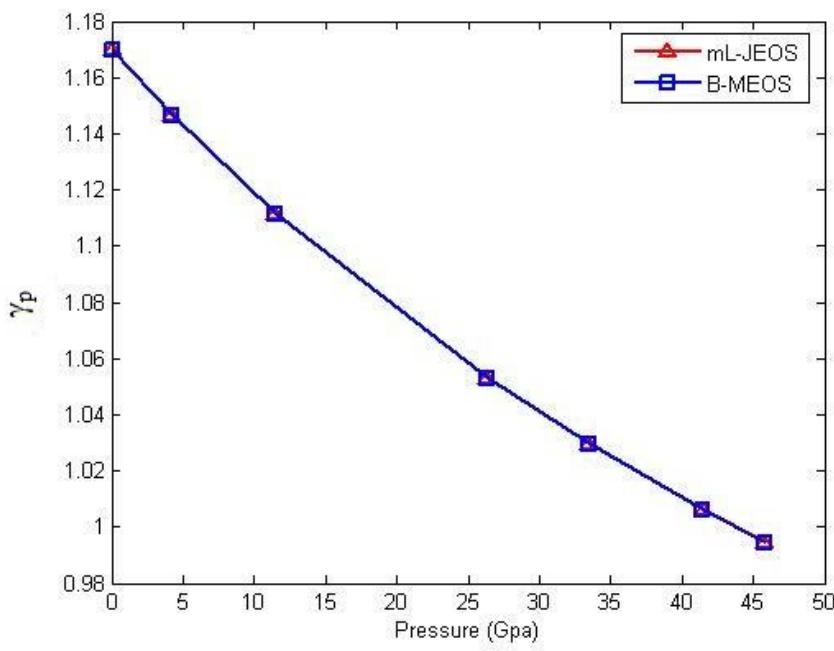

Figure 7. Variation of Grüneisen parameter with high pressure.

\subsection{Calculation of pfs, using effect of pressure on} Grüneisen parameter

Accounting the effect of pressure on " $\gamma_{\mathrm{o}}$ ", we can get the theoretical results in calculating pfs, which approach to the experiment data [16] better than in the above calculation when we assumed " $\gamma_{\mathrm{o}}$ " is pressure independent.

Figs (5\& 6) show the different results for "pfs" under high pressure using two different equations of state "B-M EOS and mL-J EOS". Combining equations (9 and 10) with with $\gamma_{P}$ in eq. (11) to get two new form of equations for evaluating $v_{P}$ and $g_{P}\left(v_{P}, V_{P}\right)$ :

$$
v_{P \gamma}=v_{0}\left(\frac{V_{P}}{V_{0}}\right)^{-\gamma_{P}}
$$

$g_{P \gamma}\left(v_{P}, V_{P}\right)=g_{0}\left(v_{0}, V_{0}\right)\left(\frac{V_{P}}{V_{0}}\right)^{-\gamma_{P}}$

Eqs.(12 and 13) are implemented to obtain improved results for analyzing pfs, under strong compression. 


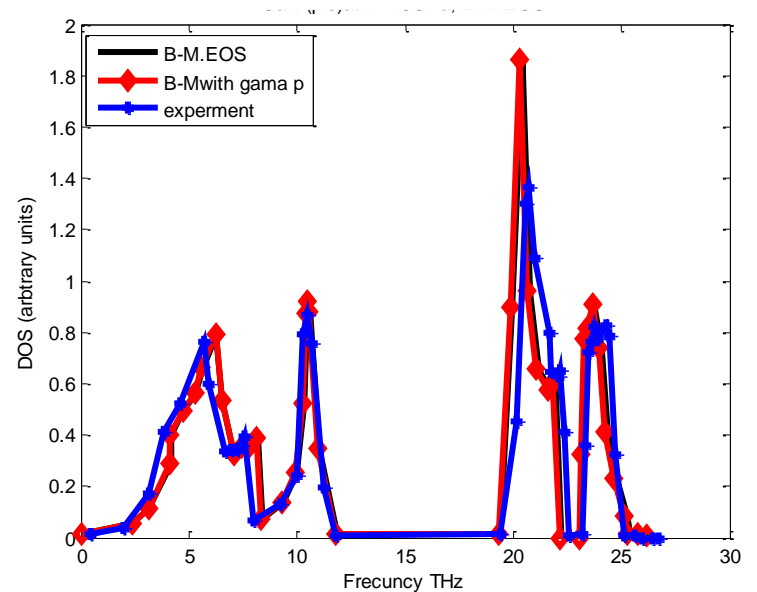

(a)

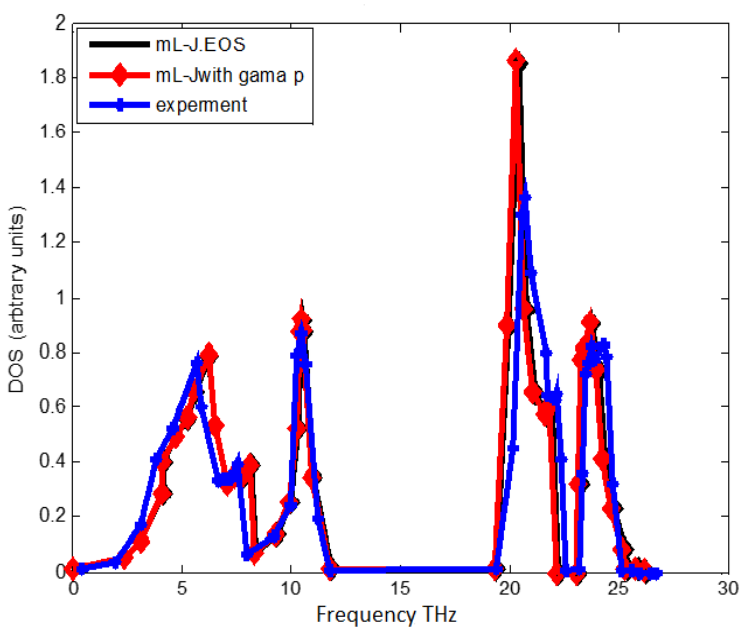

(b)

Figure 8. phonon frequency spectrum for GaN under pressure $P=15$ GPa using (a) B-M EOS (b) mL-J EOS on considering the effect of pressure on $\gamma$, and compared with experimental data [16].

\section{Discussion}

The current study implements the use two universal EOS to describe, influence of pressure the volume compression ratio, bulk modulus and phonon frequency spectrum of GaN semiconductor. The results of $\mathrm{V}_{\mathrm{p}} / \mathrm{V}_{0}$, Bulk modulus and $\gamma$ parameter, calculated with the two EOSs are viewed to be similar and the curves are fit on each other due to very small divergence of their data, over the entire pressure range up to an enormous level 45Gpa. Accordingly, the two equations of state are seen as a two identical EOSs. However, when the result of volume compression ratio from fig. 1 was combined with eq.12 and eq.13, for calculating pfs, the two EOSs have given different results as can be observed from figs. $8 \& 9$.

It is clear that the $\gamma$ pressure dependence considerations has effects on the trend of pfs variations. When $\gamma$ was assumed pressure dependent, the obtained results of pfs with the EOSs indicated in figs 8-9, were improved and fitted the experimental data better than that of figs.5-6.

By comparing of the results in the present work with experimental result [16] we got an excellent agreement for evaluation "pfs" under high pressure up to $15 \mathrm{GPa}$ by using the above two equations, but when the pressure was exceeded to $30 \mathrm{GPa}$, we got different results. Consequently, we treated this concern by assuming the effect of pressure on $\gamma$.

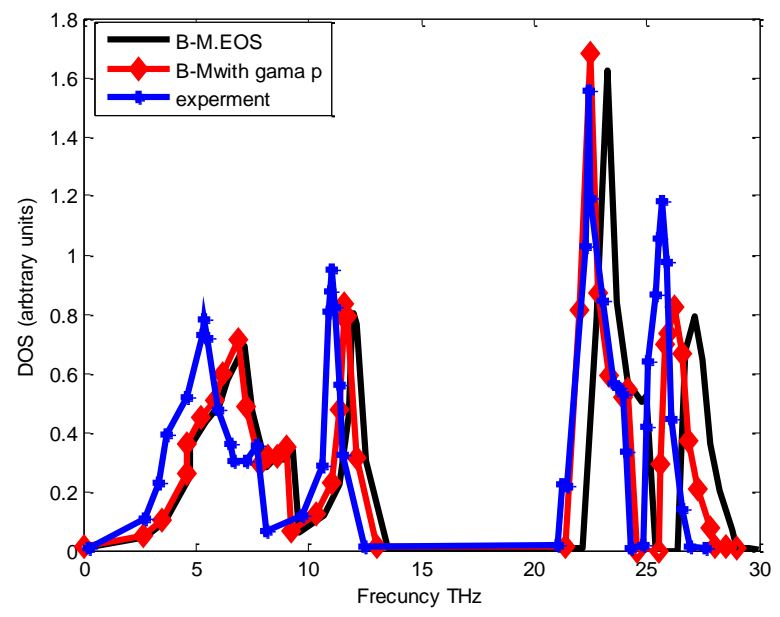

(a)

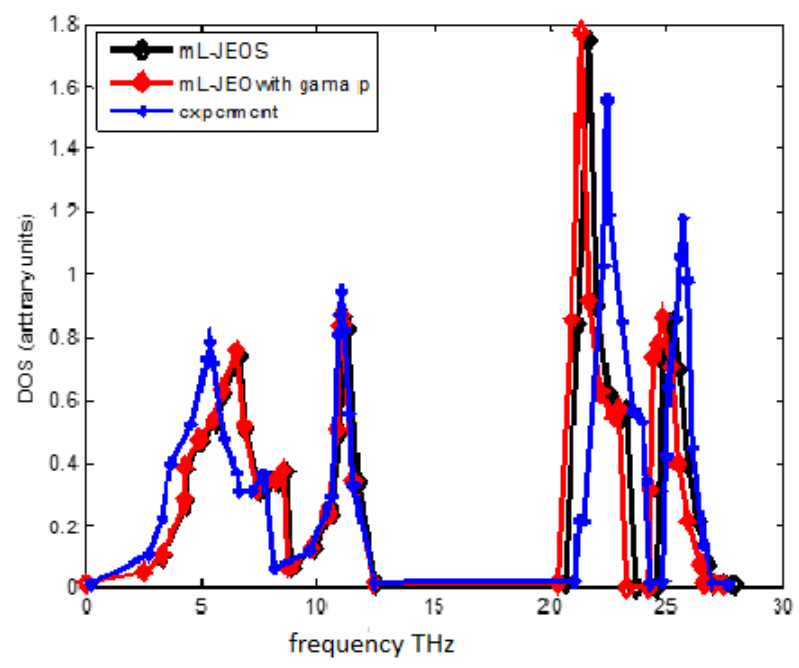

(b)

Figure 9. phonon frequency spectrum for GaN under pressure $P=30 G P a$, using (a) B-M EOS. (b) $m L-J$ EOS, where $\gamma$ is assumed pressure dependent. Compared with experimental data represented with blue line [16].

\section{Conclusion}

The variation of "pfs" for GaN has been evaluated by using two equation of state for solids "B-MEOS and mLJEOS", one time with $\gamma$ without effect of pressure Figs. $(4,5)$, and second time with effect of pressure on $\gamma$ up to 30 GPa Figs. (7,8). The present work displays that BM EOS is more suitable and give very good agreement results with (Herriman et al. 2018) compared with mL-JEOS for range of pressure up to $30 \mathrm{GPa}$ when using $\gamma_{\mathrm{p}}$, Figs. $(7,8)$, the reason of the different conclusion between results of two equations that B-M EOS based on mechanical properties of solid but mL-JEOS based on inter atomic potential.

\section{Nomenclature}

\section{GaN Gallium arsenide}

$\mathrm{V}_{\mathrm{P}} / \mathrm{V}_{0} \quad$ Volume compression ratio

$\mathrm{V}_{0} \quad$ Volume at ambient condition

$\mathrm{V}_{\mathrm{P}} \quad$ Volume under high pressure

EOS Equation of state

a Lattice parameter

$\gamma_{\mathrm{O}} \quad$ Grüneisen parameter

$\gamma_{\mathrm{P}} \quad$ Grüneisen parameter

Bo $\quad$ Bulk modulus

$\mathrm{g}_{\mathrm{o}}\left(\mathrm{v}_{\mathrm{o}}, \mathrm{V}_{\mathrm{o}}\right) \quad$ Phonon density of state at ambient condition 
$g_{P}\left(v_{P}, V_{P}\right)$

pfs

\section{References}

[1] K.M. Benali, "First-Principles study of structural, elastic and electronic properties of $\mathrm{AlN}$ and $\mathrm{GaN}$ semiconductors under pressure effect and magnetism in AlN:Mn and GaN:Mn systems," PhD. Thesis. Aboubakrbelkaiduniversity - Tlemcen faculty of sciencesPhysics department, 2004.

[2] M. Ferhat, A.Zaoui, M.Certier and B. Khelifa, “ Empirical tight- binding band structure of zinc-blende nitrides GaN, AlN, and BN," Phys. Stat. sol. (b) 195, 415,1996.

[3] S. Berrah, H. Abid, A. Boukortt and M. Sehil, "Band gap of cubic AlN, GaNandInN compounds under pressure," Turk J Phys. 30, 513-518, 2006.

[4] S.K. Jalal, A.M. Al-Sheikh, R.H. Al-Saqa, " High Pressure Effects on the Phonon Frequency Spectrum of Silicon Nanoparticle," Iran J Sci Technol Trans Sci 45, 391-396, 2021.

[5] S. K. J, S. mawood, "Size dependent thermodynamic properties of nanoparticles, "International Journal of Thermodynamics, Vol. 23 (No. 4), 245-250,2020.

[6] R. J. Angel, F. Miozzi, M. Alvaro," Limits to the Validity of Thermal-Pressure Equations of State" Minerals 9, 562,2019.

[7] F. Birch, "Finite elastic strain of cubic crystals," phys. Rev. $71 ; 809-824,1947$

[8] S. Jiuxun, “ A modified Lennard-Jones type equation of state for solids strictly satisfying the spinodal condition," J. Phys.: Condens. Matter 17; L103L111,2005.

[9] I. O. Radi; , M.A.Abdulsatter; M. Abdul-Lettif,“ Semiemperical LUC- INDO calculations on the effect of pressure on the electronic structure of diamond," Phys. Stat. Sol. b, 244(4), 1304-1317,2007.
[10] S. K. Jalal, A. M. Al-Sheikh, "heoretical High Pressure Study for Thermoelastic Properties of NaCl-B1. Raf. J. Sci., Vol. 25, No.3, pp. 80- 89, 2014.

[11] F. D. Murnaghan, "Finite deformations of an elastic solid," Am. J. Math.,49. 235- 260,1937.

[12] S. Adachi, "Properties of Group IV, III-VI and II-VI semiconductors," Wiley, N. Y., chap.2,2005.

[13] N.E.Christensen and I. Gorczyca, "Optical and structural properties of III-V nitrides under pressure," Physical Review B. 0163-1829/94/50(7)/4397(19), 1994.

[14] Xiao-wei Sun et al, "Heat Capacity and GrÄuneisen Parameter for GaN with Zinc-Blende Structure" Chinese journal of chemical physics,(20),3,2007.

[15] A. M.AL-sheikh, R. H. AL-Saqa, "Evaluation of High Pressure Effect of Phonon Frequency Spectrum for Gold by Using Different Equations of State (EOS), " Global Proceedings Repository, American Research Foundation, http://proceedings.sriweb.org ISSN 2476017X, pp 58-278, 2019.

[16] J. E. Herriman, O. Hellman, and B. Fultz, "Phonon thermo dynamics and elastic behavior of $\mathrm{GaN}$ at high temperatures and pressures," physical review B 98, $214105,2018$.

[17] R. H. AL-Saqa, S. J. AL-Taie, "Theoretical Study of Mechanical, Elastic and Phonon Frequency Spectrum Properties for GaAs at High Pressure,"Journal of Siberian Federal University. Mathematics \& Physics, 12, (3), 371-378,2019.

[18] D. L. Anderson, "Theory of the Earth. Blackwell Scientific Publications, " Oxford, UK,1989.

[19] R. Boehler; J.Ramakrishnan, "Experime ntal results on the pressure dependence of the Grüneisen parameter," A review. J. Geophy. Res., 85 (B12), 6996-7002,1980. 